\title{
Optical vortices from closed-loop subwavelength slits
}

\author{
Etienne Brasselet* \\ ${ }^{1}$ University of Bordeaux, LOMA, UMR 5798, Talence F 33400, France \\ ${ }^{2}$ CNRS, LOMA, UMR 5798, Talence F 33400, France \\ ${ }^{*}$ Corresponding author: e.brasselet@loma.u bordeaux1.fr
}

\begin{abstract}
We report on the singular shaping of light using closed loop subwavelength slits whose shape is homeomorphic to the circle. Various sets of optical phase singularities can be generated depending on the given closed path whose geometry tailors the spin orbit interaction for the light that passes through the curved slit. Here three families of closed loop curves are considered polygons, hypocycloids, and epicycloids.
\end{abstract}

OCIS codes: (050.4865) Optical vortices; (050.2555) Form birefringence; (310.6628) Subwavelength structures, nanostructures.

When light passes through a structured material endowed with subwavelength features, its propagation constant may depend on its polarization state even if all of its constituents are optically isotropic. One usually refers to form birefringence. It has often been used to realize various types of optical elements, among which are birefringent waveplates able to manipulate the polarization state of light, i.e., its spin angular momentum. The orbital angular momentum of light of a light beam, which is related to its spatial degrees of freedom, can also be controlled. This can be achieved using appropriate spatial distribution of the effective optical axis from which an output light field results with a space-variant optical phase [1].

A simple approach to the geometrical control of the spatial distribution of the phase of a light field can be grasped from Jones formalism. To this aim, let us consider a curvilinear birefringent optical element defined by a closed path $C(\phi)$ encircling the origin in the $(x, y)$ plane, parametrized by the azimuthal angle $\phi$ defined as $\tan \phi=y / x$, and characterized by a constant birefringent phase retardation $\Delta$. Moreover, let us assume that the azimuthal angle $\psi(\phi)$ of the tangent to $C$ locally defines the optical axis orientation. Then, we consider an incident circularly polarized incident plane wave propagating along the $z$ axis associated with the Jones vector $\mathbf{E}_{\text {in }}=E_{0} \mathbf{c}_{\sigma}$ where $\mathbf{c}_{\sigma}=(\mathbf{x}+i \sigma \mathbf{y}) / \sqrt{ } 2, \sigma= \pm 1$, refers to the circular polarization basis. Just after the optical element, the output light field expresses along $C$ as $\mathbf{E}_{\text {out }}(\phi)=E_{0}\left[\cos (\Delta / 2) \mathbf{c}_{\sigma}+i \sin (\Delta / 2) e^{i 2 \sigma \psi(\phi)} \mathbf{c}_{-\sigma}\right]$.

When $C$ is a circle, $\psi(\phi)=\phi+\pi / 2$, and the contracircularly polarized output light field component has an azimuthal dependence of the amplitude of the form $e^{i 2 \sigma \phi}$, which is associated with an optical vortex field carrying on-axis optical phase singularity with topological charge $\ell= \pm 2$. In practice, this corresponds to the case of a circular subwavelength slit, which locally behaves as a birefringent retarder whose main axes are directed parallel and perpendicular to it [2] , as recently reported experimentally [3]. In contrast, when the axial symmetry of the circuit $C$ is broken, the generation of a singular optical field carrying a charge-two vortex with a broad orbital angular momentum spectrum has been predicted [3].

Here we show that the nature of the optical phase singularities that result from the diffraction of light on two-dimensional closed-loop slits homeomorphic to the circle (i.e., obtained from continuous deformation of a circle) can, in fact, differ from previous predictions [3]. This is done by introducing three different kinds of path $C(\phi)$. The first one consists of regular convex polygons $(p)$ with $m_{p}$ sides, $m_{p} \geq 3$. The two others, hypocycloids $(h)$ and epicycloids $(e)$, correspond to the trajectory of a point attached to a given circle as it rolls without slipping within and around another fixed circle, respectively. By considering these curves to be inscribed in the unit circle, the parametrization of the hypocycloid expresses as

$$
\begin{aligned}
& x(\theta)=\frac{m_{h}-1}{m_{h}} \cos \theta+\frac{1}{m_{h}} \cos \left[\left(m_{h}-1\right) \theta\right], \\
& y(\theta)=\frac{m_{h}-1}{m_{h}} \sin \theta-\frac{1}{m_{h}} \sin \left[\left(m_{h}-1\right) \theta\right],
\end{aligned}
$$

with $m_{h}$ integer, $m_{h} \geq 3$ and $0 \leq \theta \leq 2 \pi$, whereas epicycloids are described by

$$
\begin{aligned}
& x(\theta)=\frac{m_{e}+1}{m_{e}+2} \cos \theta-\frac{1}{m_{e}+2} \cos \left[\left(m_{e}+1\right) \theta\right], \\
& y(\theta)=\frac{m_{e}+1}{m_{e}+2} \sin \theta-\frac{1}{m_{e}+2} \sin \left[\left(m_{e}+1\right) \theta\right],
\end{aligned}
$$

with $m_{e}$ integer, $m_{e} \geq 1$. The circle is referred to as the special case $m_{e}=0$. Note that inscription into the unit circle of the epicycloid with $m_{e}=1$ requires applying the transformation $(x, y) \rightarrow(2 / \sqrt{ } 3)(x+1 / 6, y)$, to Eq. (2). Moreover, note that the variable $\theta$ should not be confused with the azimuthal angle $\phi$.

The proposed choice of geometries is motivated by the following considerations. First we note that a polygon tends to the circle as $m_{p}$ increases and the role of discrete rotational symmetry on the generated phase singularities at finite value of $m_{p}$, if any, is an issue that we aim at addressing. Second, hypocycloids and epicycloids are purposely chosen regarding their topological features. Indeed, following previous Jones considerations, the output field component (just after the slit), whose circular polarization state is orthogonal to the incident one, acquires an azimuthal dependence of the amplitude of the form $e^{i 2 \sigma \psi_{j}(\phi)}$, with $j=(h, e)$, where $\ell_{j}=(1 / 2 \pi) \oint \mathrm{d} \psi_{j}$, is an integer. This leads us to introduce the topological charges 


$$
\ell_{h}=\sigma\left(2-m_{h}\right)
$$

and

$$
\ell_{e}=\sigma\left(2+m_{e}\right)
$$

associated with the singular near-field emerging from the hypocycloidal and cycloidal slits, respectively. That is to say, controlled topological shaping of light from closed-loop slits can be achieved in the near field. How such singular shaping of light is transported into the far field by mere propagation is another issue addressed hereafter.

The far-field electric field is calculated in the framework of scalar Fraunhofer diffraction theory and the aperture plane is assumed to be perfectly opaque outside the slit. In addition, we restrict our analysis to the component of the output light field whose polarization state is $\mathbf{c}_{-\sigma}$ and whose amplitude is given, up to a constant multiplying factor that characterizes the polarization conversion efficiency, by

$$
E_{-\sigma}(\xi, \eta) \propto \iint t(x, y) e^{-i 2 \pi \frac{R}{\lambda}(\xi x+\eta y)} \mathrm{d} x \mathrm{~d} y,
$$

where $t=\exp \left(i 2 \sigma \psi_{j}\right), j=(p, h, e)$, is the complex transmittance that accounts for the particular geometry of the closed-loop slit of width $w$, inscribed in a circle of radius $R$, and $\lambda$ is the wavelength. Here, $x$ and $y$ are the reduced coordinates with respect to $R$, as they appear in Eqs. (1) and (2), whereas $\xi$ and $\eta$ are the reduced reciprocal coordinates, which vary between -1 and 1 . More precisely, the following expressions can be derived for the transmittance phase $\Phi_{j}(\phi)=2 \sigma \psi_{j}(\phi): \Phi_{p}^{(n)}=2 \sigma \pi[(2 n-$ $\left.1) / m_{p}-1 / 2\right]$ for the $n$th side of the polygon, $\left(1 \leq n \leq m_{p}\right), \quad \Phi_{h}(\phi)=\ell_{h} \theta_{h}(\phi)$ and $\Phi_{e}(\phi)=\ell_{e} \theta_{e}(\phi)$ [note that $\theta_{h}(\phi) \neq \theta_{e}(\phi)$ ]. In what follows, results obtained for the three families of geometries are discussed by using $\sigma=1, w / R=0.05$ and $\lambda=500 \mathrm{~nm}$, with $R=10 \mu \mathrm{m}$.

Polygonal slits. Far-field intensity and phase for the first three polygons with $m_{p}=(3,4,5)$, are shown in Fig. 1. Although on-axis intensity is zero in all cases, it is associated with a spiraling azimuthal profile of the phase only for $m_{p} \neq 4$. Indeed, as shown in the third row of Fig. 1, azimuthal phase profiles around the origin, taken along a circle that passes by local intensity maxima (see dashed curve on intensity profiles), have a spiraling behavior associated with the topological charge -1 for $m_{p}=3$ and +2 for $m_{p} \geq 5$, whereas $\pi$ phase dislocations appear for $m_{p}=4$. This demonstrates that polygonal slits can be used to generate optical vortex beams with topological charges \pm 1 and \pm 2 , whose sign is controlled by the handedness of the incident circular polarization state, $\sigma= \pm 1$.

Hypocycloidal slits. A richer topological diversity is obtained when using hypocycloidal slits rather than polygonal ones. This is shown in Fig. 2 where spiraling phase patterns around the origin are associated with topological charges $-1,-2$, and -3 for $m_{h}=(3,4,5)$, respectively. Such a far-field behavior thus follows the near-field topological features given by Eq. (3). This
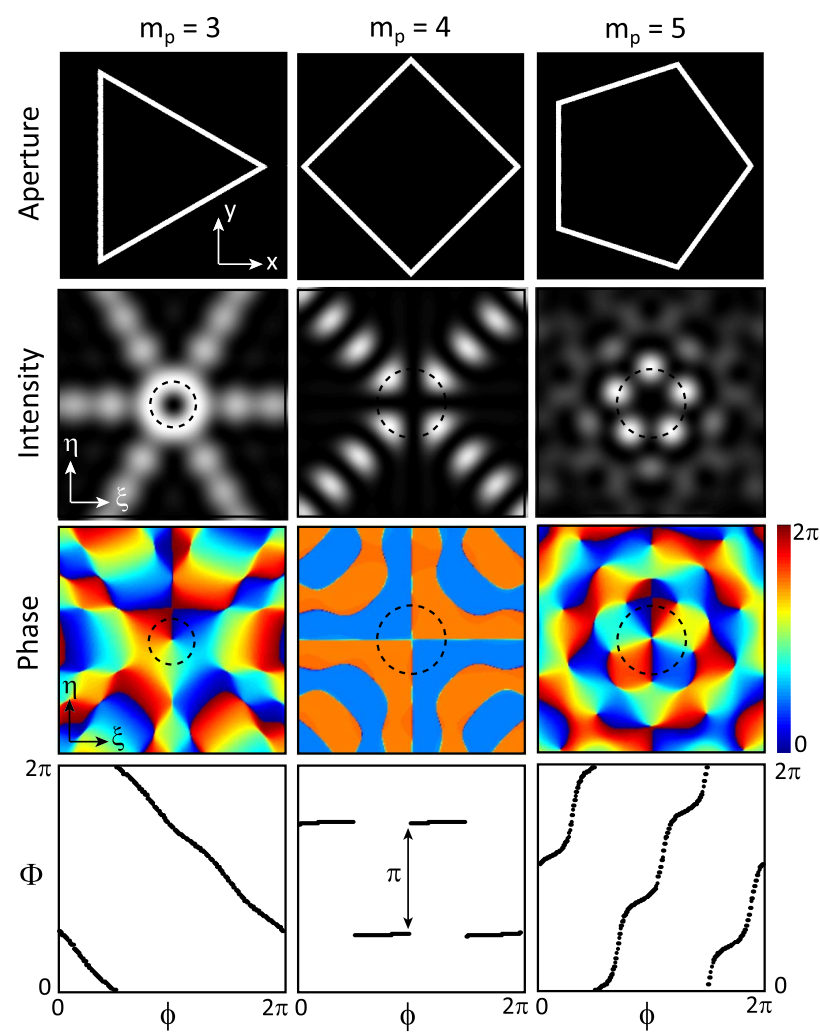

Fig. 1. First row: slit aperture for polygons with $m_{p}=3$ (triangle), 4 (square), and 5 (pentagon). Second and third rows: Fraunhofer diffraction intensity and phase patterns in the reciprocal coordinate system, here displayed in the range

$0.1 \leq(\xi, \eta) \leq 0.1$. Fourth row: azimuthal dependence of the phase along the closed contours that appear as dashed curves on phase plots.

illustrates quantitatively the topological shaping abilities of closed-loop slits with appropriate design, which cannot be achieved using circular slits [3]. However, despite being quantized, we note that obtained helical phase profiles have a nonuniform azimuthal dependence, which is reminiscent of the nonuniform near-field azimuthal phase dependence $\Phi_{h}(\phi)$.

Epicycloidal slits. Although the behavior of epicycloidal slits with $m_{e}>0$ quantitatively departs from the circular case $m_{e}=0$, see Fig. 3 , the circulation of the phase around a circle path nearby the propagation direction always gives a topological charge of two (see white dashed circles in Fig. 3) in contrast to the case of hypocycloids. On-axis higher-order topological diversity indeed does not follow Eq. (4) for epicycloidal slits. Nevertheless, the signature of the near-field topological charge given by Eq. (4) is recovered by considering closed circuits passing through the main maxima of intensity, see black dashed circuits in Fig. 3 for $m_{e}=1$ and $m_{e}=2$. On this ground, the epicycloidal design appears less interesting than the hypocycloidal one.

Higher-order slits. One may argue that all structures should eventually behave as a circular slit for large enough indices $m_{j}, j=(p, h, e)$, which is illustrated in Fig. $\underline{4}$ where $m_{j}=8$. In that case, all designs tend to give on-axis phase singularity with topological charge $2 \sigma$. Still, we notice that the phase patterns have distinct fine 

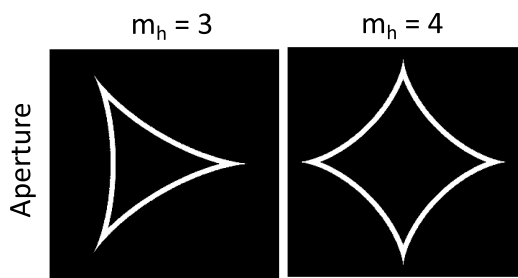

$m_{h}=5$
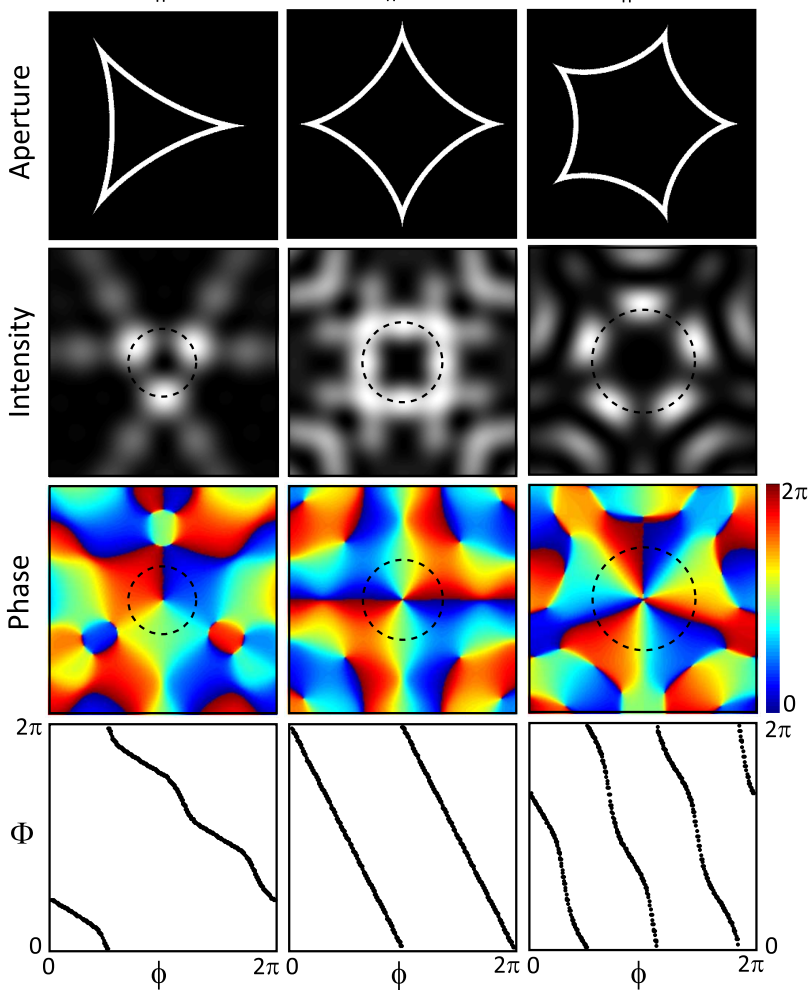

Fig. 2. Same as Fig. 1 for hypocycloids with $m_{h}=3$ (deltoid), 4 (astroid), and 5.
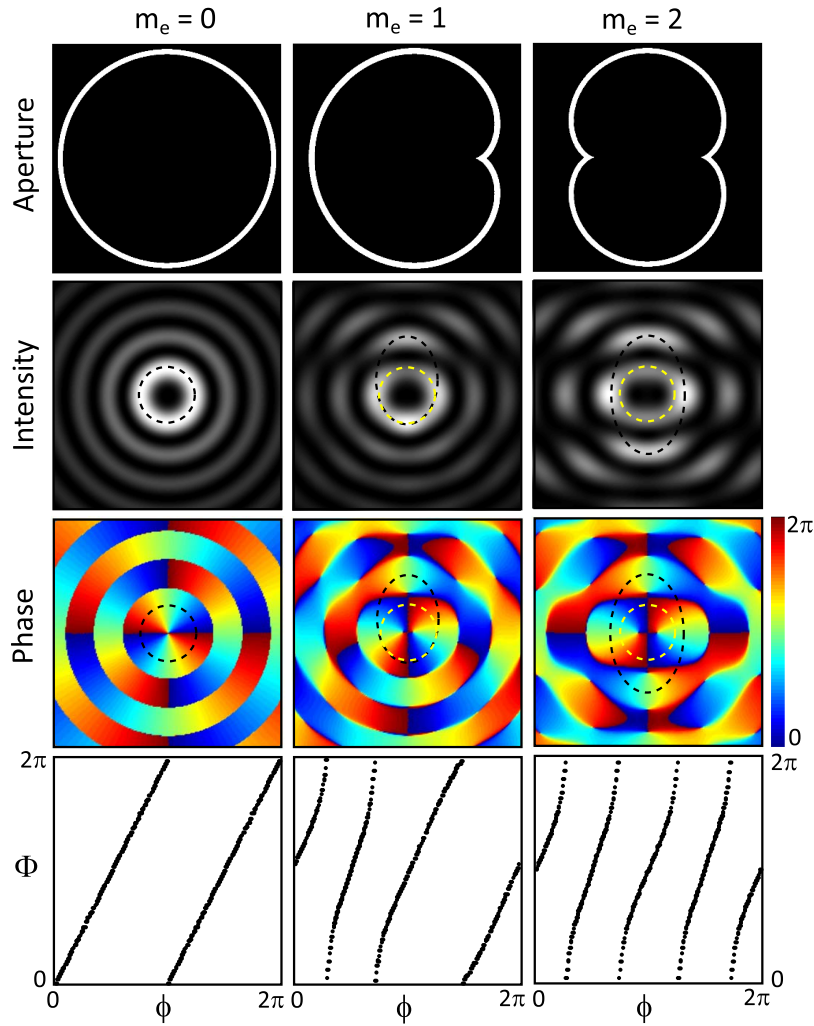

Fig. 3. Same as Fig. 1 for epicycloids with $m_{e}=0$ (circle), 1 (cardiod), and 2. Bottom row: phase profiles refers to elliptical path indicated by black dashed ellipse on corresponding upper rows.
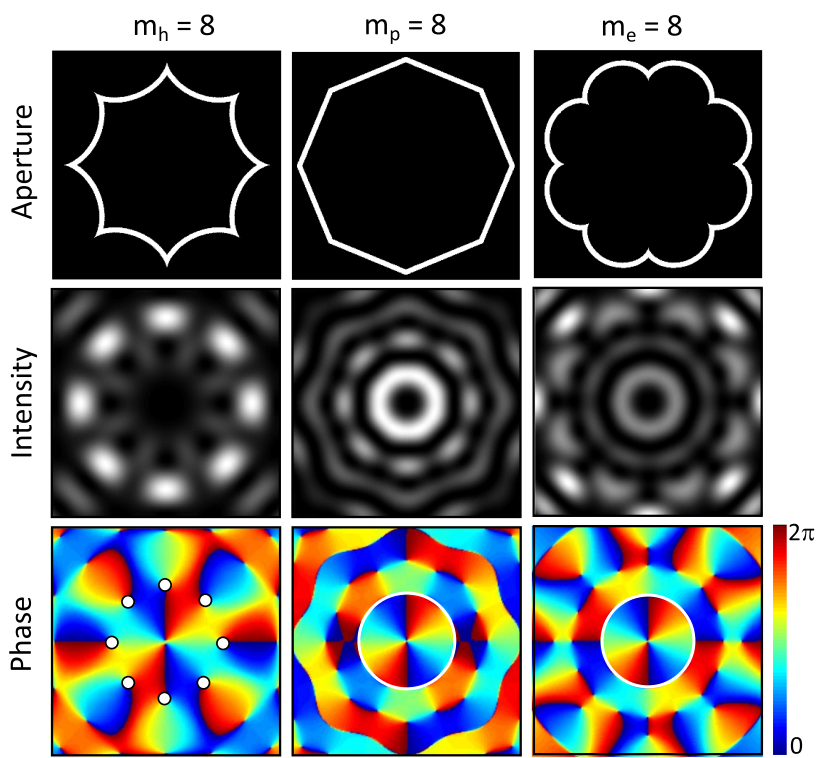

Fig. 4. Comparative analysis of higher order structures with $m_{i}=8$ and $i=(h, p, e)$. Bottom row: see text for details. Coordinate ranges are similar to those of Fig. 1 .

structures depending on the design of the slit at finite values of $m_{j}$. For instance, $m_{h}$ phase singularities with topological charge $-\sigma$ encircle the origin in the hypocycloidal case (see circle markers in Fig. 4) whereas a quasi-dislocation ring is observed for both the polygonal and epicycloidal cases (see white circles in Fig. 4).

Finally, we note that the presence of a constellation of unit charge optical phase singularities, regardless of the geometry (see Fig. 4), is a consequence of the broken axial symmetry when $C$ differs from the circle. Vortex splitting is indeed a general feature of higher-order singularities that are intrinsically unstable $[4,5]$.

To conclude, appropriate designs of closed-loop slits enable topological shaping of light via spin-orbit interaction of light. Three kinds of geometries have been proposed and analyzed: polygons, hypocycloids, and epicycloids. The generation of various sets of optical vortices have been shown and allow us to envision a novel route to realize spin-orbit interfaces able to manipulate the orbital angular momentum of light at a small spatial scale.

\section{References}

1. G. Biener, A. Niv, V. Kleiner, and E. Hasman, Opt. Lett. 27, 1875 (2002).

2. P. F. Chimento, N. V. Kuzmin, J. Bosman, P. F. A. Alkemade, G. W. 't Hooft, and E. R. Eliel, Opt. Express 19, 24219 (2011).

3. P. F. Chimento, P. F. A. Alkemade, G. W. 't Hooft, and E. R. Eliel, Opt. Lett. 37, 4946 (2012).

4. M. S. Soskin, V. N. Gorshkov, M. V. Vasnetsov, J. T. Malos, and N. R. Heckenberg, Phys. Rev. A 56, 4064 (1997).

5. V. G. Denisenko, A. Minovich, A. S. Desyatnikov, W. Krolikowski, M. S. Soskin, and Y. S. Kivshar, Opt. Lett. 33, 89 (2008). 